\title{
Anabases
}

ANABASES Traditions et réceptions de l'Antiquité

17 | 2013

Varia

\section{Sparta e Atene tra Francia e Prussia: da Benjamin Constant a Georg Busolt (1804-1878)}

Carmine Pisano

\section{(2) OpenEdition}

Journals

Edizione digitale

URL: http://journals.openedition.org/anabases/4079

DOI: 10.4000/anabases.4079

ISSN: 2256-9421

Editore

E.R.A.S.M.E.

\section{Edizione cartacea}

Data di pubblicazione: 1 marzo 2013

Paginazione: 11-25

ISSN: 1774-4296

\section{Notizia bibliografica digitale}

Carmine Pisano, « Sparta e Atene tra Francia e Prussia: da Benjamin Constant a Georg Busolt (1804-1878) », Anabases [Online], 17 | 2013, Messo online il 01 avril 2016, consultato il 20 octobre 2019. URL : http://journals.openedition.org/anabases/4079 ; DOI : 10.4000/anabases.4079

(c) Anabases 
Anabases 17 (2013), p. 11-25.

\section{Sparta e Atene tra Francia e Prussia: da Benjamin Constant a Georg Busolt (1804-1878)}

Carmine Pisano

In UnA ReCente Conferenza tenuta all'Università di Napoli Federico II, Pascal Payen ha analizzato i fattori che hanno contribuito alla «costituzione della storia come scienza nel XIX secolo», evidenziando il ruolo svolto dai «modelli antichi» nella definizione dello statuto epistemologico della nuova disciplina ${ }^{1}$. Nata in Germania nei primi anni dell'Ottocento nell'ambito dell' Altertumswissenschaft in piena formazione, la moderna scienza storica si costituisce, in opposizione alla cultura francese, come reazione al trauma dell'occupazione napoleonica (1806-1808). La sua nascita - osserva Payen - è strettamente legata alle "guerre di liberazione» combattute contro l'invasore francese e alla rifondazione dell'Università di Berlino (1809-1810): in breve, alla ripresa della

1 P. PAYEN, " La constitution de l'histoire comme science au XIX ${ }^{\mathrm{e}}$ siècle et ses modèles anciens ", Università degli Studi di Napoli Federico II, 18-06-2010. La nuova Università prussiana, fondata per iniziativa di Wilhelm von Humboldt e Johann Gottlieb Fichte, promuove un programma educativo in cui gli studi storico-filologici assurgono al rango di Bildungsfundament (P. Berglar, Wilhelm von Humboldt, Hamburg, Rowohlt, 1970, p. 90), dando avvio a quella che Carlo Antoni (La lotta contro la ragione, Firenze, Sansoni, 1942, p. 154) definisce la «tirannia della Grecia sulla Germania». È in questo contesto che lo studio dei classici diviene elemento portante dell'identità tedesca: «Il meglio dello spirito greco - scriverà più tardi Ulrich von Wilamowitz - può comprenderlo solo chi sia di razza germanica» (L. CAnfora, Cultura classica e crisi tedesca, Bari, De Donato, 1977, p. 159-160). 
Prussia umiliata. Sono gli storici antichi e Tucidide, in particolare, a fungere da modello per l'elaborazione della nuova disciplina storica ${ }^{2}$. Ma perché proprio Tucidide?

Payen evidenzia il recupero da parte dei primi storici tedeschi di due aspetti essenziali della concezione tucididea: l'opera storica intesa come «acquisizione per sempre» e l'impegno politico dello storico. In quanto ktêma es aié, l'opera tucididea si presenta come strumento di riflessione e di scienza politica, offerto alle generazioni future. È da questa idea che la nascente Altertumswissenschaft ricava la concezione della storia come "conoscenza di quel che è accaduto» (Wissen von dem Geschehenen). Si tratta di una forma di conoscenza che consente di pensare e affrontare i problemi politici del presente. Per gli storici tedeschi, come già per Tucidide, fare storia significa agire politicamente. Forte di queste osservazioni, Payen conclude a giusto titolo che è la duplice dimensione gnoseologica e politica dell'attività storiografica a fare di Tucidide un modello «attuale» per la nascente storiografia tedesca.

Nella Prussia appena liberata dalle armi francesi, ad apparire «attuale» non è solo la concezione storica di Tucidide ma anche il contenuto della sua opera. Nel periodo compreso tra la Quarta coalizione (1806-1807) e la Guerra franco-prussiana (18701871), «la guerra dei Peloponnesiaci e degli Ateniesi» diviene modello delle controversie politiche e scientifiche che oppongono Francia e Prussia e che assumono nella storiografia contemporanea i connotati ideologici del conflitto tra Atene e Sparta. Il fenomeno è stato studiato da Pierre Vidal-Naquet e Nicole Loraux nel contesto della storiografia francese ${ }^{3}$. Il nostro obiettivo è valutare in che misura e in che modo mutano dalla Francia alla Germania - contesti in cui l'interesse per Tucidide matura più o meno contemporaneamente - l'analisi della Guerra del Peloponneso e la rappresentazione dei modelli statuali antichi. In altri termini, se nella cultura francese l'opposizione alla Prussia si presenta nei termini del conflitto tra "un'Atene borghese e letterata» e «una Sparta virtuosa e militare ${ }^{4}$, si tratta di definire quali valori socio-politici sono associati dalla storiografia prussiana alle "repubbliche» greche, ovvero di analizzare come gli intellettuali francesi e tedeschi rileggono e risemantizzano le categorie di "spartano»e di «ateniese» nell'ambito del dibattito politico-culturale del XIX secolo.

2 Su usi e riletture di Tucidide in epoca moderna (secoli XV-Xx), cf. i contributi raccolti in V. Fromentin - S. Gotteland - P. Payen (éd.), Ombres de Thucydide: la réception de l'historien depuis l'Antiquité jusqu'au début du XXe siècle, Pessac, Ausonius Éditions, 2010, p. 495-742.

3 N. Loraux, P. VidAL-NaqueT, «La formazione dell'Atene borghese. Saggio di storia della storiografia 1750-1850", in P. VIDAL-NAQUET, La democrazia greca nellimmaginario dei moderni, trad. it. Milano, Il Saggiatore, 1996, p. 165-218.

4 P. VidAL-NAQUET, «La Grecia nell'immaginario degli uomini della Rivoluzione», in ID., La democrazia greca, p. 222; cf. anche A.M. IACONO, L'utopia e i Greci, in S. SETTis (a cura di), I Greci. Storia, cultura, arte, società, I, Noi e i Greci, Torino, Einaudi, 1996, p. $889-890$. 
Su una pagina del proprio Journal intime datata maggio $1804^{5}$, l'intellettuale liberale Benjamin Constant (1767-1830) mette in guardia dalle teorie politiche del filosofo tedesco Johann Gottlieb Fichte, presentate qualche anno prima in uno scritto intitolato Lo Stato commerciale chiuso (1800). Benché il modello di Stato tratteggiato da Fichte derivi dalle «migliori intenzioni del mondo», si tratta, infatti, di «idee spartane», simili a quelle che avevano portato Robespierre al potere. Ma che cosa ha di «spartano» il modello politico di Fichte? Il filosofo tedesco prospetta una società divisa in tre classi (produttori, costruttori e commercianti), la cui cooperazione e uguaglianza è garantita dalla chiusura dello Stato nei confronti di influenze economiche esterne. Attraverso una serie di misure rigorose, lo Stato assicura un'equa distribuzione di beni e proprietà tra i cittadini, che sono liberi di pensare e vivere come meglio credono. Nella definizione del suo modello politico Fichte non sembra avere in mente Sparta: convinto che lo Stato razionale debba ancora venire, il filosofo guarda con poca attenzione ai modelli antichi. In Fondamenti del diritto naturale (1796), Fichte precisa anzi che gli efori di cui fa menzione non hanno nulla in comune con l'omonima magistratura spartana ${ }^{6}$. Alla luce della posizione di Fichte, occorre chiedersi che cosa Constant, giudicando lo Stato commerciale chiuso, intenda per «spartano», ovvero quale significato assuma questo aggettivo nella cultura liberale francese dei primi anni dell'Ottocento.

È lo stesso Constant a fornirci un indizio prezioso nel momento in cui accosta le idee di Fichte a quelle di Robespierre e seguaci. Sparta era stata il grande modello «repubblicano» degli illuministi francesi (Montesquieu, Rousseau, Mably) e dei capi della Rivoluzione, in particolare dei dirigenti montagnardi ${ }^{7}$. Durante il periodo in cui si trovano al potere (giugno 1793 - luglio 1794), Sparta, la città degli «Eguali» simile a "una grande luce che brilla tra tenebre immense», compare nei discorsi di Robespierre e Saint-Just come modello di società «trasparente», fondata sui valori civili della «libertà» e della «virtù» ${ }^{8}$. Per i capi montagnardi Sparta significa uguaglianza e

5 J. Mistler (éd.), Benjamin Constant. Journal intime, Monaco, Éditions du Rocher, 1945, p. 183.

6 Sul modello statuale fichtiano, cf. E. Rawson, The Spartan Tradition in European Thought, Oxford, Clarendon Press, 1969, p. 295; 317-318.

7 Occorre, tuttavia, ricordare che non tutti gli illuministi francesi condivisero la «spartomania» allora in voga. Tra i partigiani di Atene vi furono personaggi del calibro di Voltaire e del barone d'Holbach, che nella città attica riconobbero il simbolo del commercio e della libertà di pensiero. Sull'uso dei modelli repubblicani antichi nella cultura illuministica, cf. G. Cambiano, «Montesquieu e le antiche repubbliche greche», Rivista di Filosofia 65 (1974), p. 93-144; ID., "Comparazioni e modelli nelle immagini settecentesche dell'antichità», Opus 6-8 (1987-1989), p. 219-236; C. Mossé, L'Antiquité dans la Révolution francaise, Paris, Albin Michel, 1989; P. Boutry - J. Raspi Serra (a cura di), La Grecia Antica. Mito e simbolo per l'età della Grande Rivoluzione, Milano, Guerini, 1991.

8 Cf. L. Canfora, Ideologie del Classicismo, Torino, Einaudi, 1980, p. 11-19. Sul concetto di «trasparenza», si veda N. Loraux, «Aux origines de la démocratie. Sur la transparence démocratique», Raison présente 49 (1979), p. 3-13. 
rigore rivoluzionario. Ma non sono certo questi i valori che il liberale Constant associa all'aggettivo «spartano». Dalla Repubblica di Robespierre alla pagina del Journal intime, la situazione politica e culturale francese muta radicalmente. La crisi degli ideali rivoluzionari e la caduta di Robespierre il 9 Termidoro anno II (27 luglio 1794) comportano profondi rivolgimenti politici che non mancano di ripercuotersi nel campo della riflessione storica e filosofica. Gli intellettuali di orientamento liberale demoliscono a poco a poco il mito di Sparta, sottoponendo a durissimi attacchi i fondamenti stessi del laconismo montagnardo. Feroci critiche sono rivolte, in primo luogo, al presunto egalitarismo spartano e alla costituzione di Licurgo. Già in pieno Terrore - siamo nel 1794 - Camille Desmoulins osserva che Licurgo non ha fatto altro che «imporre privazioni ai suoi concittadini, rendendoli uguali come la tempesta rende uguali tutti i naufraghi». Come prova la libertà di critica goduta da Aristofane, «i veri repubblicani [...] per principio e per istinto erano gli Ateniesi», garanti della democrazia e del «diritto di sbagliarsi» (l'odierna libertà di stampa). Sono pertanto la disciplina egalitaria dell'esercito ateniese, le leggi di Solone e la «clemenza di Trasibulo» che la Francia dovrebbe tenere a modello?

Nella campagna anti-montagnarda condotta da Desmoulins sulle pagine di Le Vieux Cordelier ${ }^{10}$, l'opposizione alla Sparta di Robespierre si manifesta nei termini di una difesa dei principi «democratici» ateniesi. La critica dei modelli antichi si fa, invece, più radicale nell'opera dell'intellettuale termidoriano Constantin Volney (1757-1820). In una celebre lezione tenuta all'École Normale poco dopo la caduta di Robespierre, Volney afferma che i Giacobini, nel loro fanatismo per la Repubblica spartana, hanno perso di vista i caratteri fondamentali della democrazia antica, aperta a un numero ristretto di cittadini liberi e fondata sul lavoro servile di enormi masse di schiavi: «Su circa quattro milioni di anime che dovettero popolare la Grecia antica, gli schiavi erano più di tre milioni.» Alla massiccia presenza di schiavi le repubbliche antiche associano una vistosa diseguaglianza sociale, accettata come «dogma» tanto dalle "popolazioni» quanto dai «legislatori». Volney si fonda sull'autorità degli storici antichi, di Tucidide in particolare, per demistificare il mito giacobino-montagnardo dell'egalitarismo spartano $^{11}$. L'interesse per il grande storico ateniese si accompagna alla denuncia dei "confronti viziosi» e delle pratiche «falsamente imitative» che avevano caratterizzato gli anni della Rivoluzione.

La denuncia dell'imitazione viziosa dei modelli antichi rappresenta la base non solo del pensiero di Volney, ma di tutta la riflessione storico-politica del periodo post-

9 Sul pensiero politico di Desmoulins, cf. P. VIDAL-NAQUET, «Lumières de la cité grecque», in ID., Les Grecs, les historiens, la démocratie: le grand écart, Paris, La Découverte, 2000, p. 187-188.

10 Il giornale, pubblicato tra il 5 dicembre 1793 e il 25 gennaio 1794, fu censurato quando non era stato ancora pubblicato il settimo numero; Desmoulins fu ghigliottinato il 5 aprile 1794.

11 Cf. Loraux, Vidal-NaQuet, «La formazione dell'Atene borghese», p. 179-182. 
termidoriano. Caduta la dittatura montagnarda, gli intellettuali liberali rivalutano la democrazia ateniese a discapito del modello spartano, la cui «imitazione» è giudicata responsabile del Terrore ${ }^{12}$. Negli Études d'histoire ancienne (1811), Pierre-Charles Levesque (1736-1812), autore nel 1795 della prima traduzione francese di Tucidide, presenta gli Spartani come «ignoranti, feroci e villani», dediti esclusivamente al mestiere delle armi: «Si ammira Lacedemone guerriera - dichiara - ma si ama la sua rivale.» Atene, la grande rivale di Sparta, appare rispettosa della proprietà e della vita privata, promotrice del commercio e dell'industria ${ }^{13}$. L'opposizione tra lo Stato laconico e quello attico, declinata nelle forme del pensiero politico termidoriano, getta le basi del grande mito dell'Atene «liberale». La riflessione di Benjamin Constant è decisamente rappresentativa di questa tendenza.

Nella celebre conferenza De la liberté des Anciens comparée à celle des Modernes, Constant riconosce la base schiavistica della democrazia antica e la distanza che separa la libertà degli antichi da quella dei moderni, ma non manca di esprimere la sua preferenza per Atene rispetto a Sparta: «Si godeva, ad Atene, di una libertà individuale molto più grande che a Sparta, perché Atene era al tempo stesso guerriera e commerciale, mentre Sparta era esclusivamente guerriera ${ }^{14}$.» Nel sistema teorico di Constant, l'antitesi Sparta/Atene riflette l'opposizione tra spirito di conquista e spirito di commercio:

12 Nel periodo post-termidoriano il «miraggio spartano» resta forte negli ambienti reazionari di estrema destra. Il conte Joseph de Maistre, uno dei più famosi esponenti del movimento contro-rivoluzionario, pubblica nel 1797 le Considérations sur la France. Da buon teorico della Restaurazione, de Maistre sostiene la necessità di un immediato ripristino della monarchia ereditaria in Francia e condanna la Rivoluzione in quanto distruzione dell'ordine politico-sociale voluto direttamente da Dio. In questo progetto di stampo chiaramente reazionario è di nuovo Sparta a fungere da modello per il presente. In polemica con Constant, de Maistre sostiene che Solone avrebbe creato la costituzione "più fragile dell'antichità», fondata sulla pratica deliberativa e la volubilità della folla. Di contro, «'istituzione più vigorosa dell'antichità profana fu quella di Lacedomone», garanzia di ordine sociale e di resistenza alle rivoluzioni. Sul programma politico-ideologico di de Maistre, cf. R. Triomphe, Joseph de Maistre. Étude sur la vie et la doctrine d'un matérialiste mystique, Genève, Droz, 1968.

13 Su Levesque, cf. P. Vidal-Naquet, «Tradition de la démocratie grecque», in M.I. Finley, Démocratie antique e démocratie moderne, trad. fr. Paris, Payot, 1976, p. 34-35.

14 Sul confronto Atene/Sparta, si veda B. Constant, Della libertà degli antichi paragonata a quella dei moderni, in ID., Principi di politica, a cura di U. CERroni, Roma, Editori Riuniti, 1970, p. 228-230. Sulla contrapposizione tra libertà degli antichi e libertà dei moderni, cf. L. Guerci, Libertà degli antichi e libertà dei moderni: Sparta, Atene e i "philosophes" nella Francia del Settecento, Napoli, Guida, 1979; F. HarTOG, "Liberté des Anciens, liberté des Modernes: la Révolution francaise et l'Antiquité», in R.P. Droit (éd.), Les Grecs, les Romains et nous. L'Antiquité est-elle moderne?, Paris, Le Monde Éditions, 1991, p. 119-138; W. Nippel, "Antike und moderne Freiheit», in W. Jens - B. Seidensticker (Hrsg.), Ferne und Nähe der Antike. Beiträge zu den Künsten und Wissenschaften der Moderne, Berlin, de Gruyter, 2003, p. 49-68. 
il primo, tipico di Sparta, dà luogo sul piano politico alla partecipazione diretta e alla libertà antica; il secondo, incarnato da Atene, porta al principio rappresentativo e alla libertà moderna ${ }^{15}$. Per Constant Atene è sinonimo di repubblica mercantile e prototipo del moderno Stato borghese-liberale: «Atene ci somiglia troppo ${ }^{16}$.» $\mathrm{Al}$ «carattere interamente moderno degli Ateniesi» è contrapposta Sparta, "questo vasto convento", dedito esclusivamente all'esercizio della guerra, estraneo a qualsiasi forma di commercio o industria. Nel panorama culturale di Constant, ovvero nella cultura liberale francese dei primi anni dell'Ottocento, Sparta evoca, in antitesi ad Atene, uno Stato assimilabile a un "convento" chiuso, guerriero ed egalitario nel senso - stabilito da Desmoulins del «naufragio comune» delle libertà individuali. Sono queste le caratteristiche del modello statuale fichtiano che Constant etichetta come «idee spartane».

L'immagine di Sparta appena analizzata è molto interessante anche per un altro motivo. Abbiamo visto come la figura «conventuale» della città laconica si delinei nella riflessione degli intellettuali termidoriani a partire dalla riscoperta di Tucidide, la cui prima traduzione francese appare nel 1795 per mano di Levesque. Nella prospettiva del traduttore, Tucidide appare come maestro di arte politica, ovvero come l'autore che "più di ogni altro deve essere studiato nei paesi in cui i cittadini potranno avere parte, un giorno, al governo». Si tratta di un'affermazione di importanza cruciale. Sin dal primo imporsi di Tucidide sulla scena culturale francese - negli anni della Rivoluzione l'autore greco più letto e studiato era stato Plutarco ${ }^{17}$ - la ricezione della sua opera storica appare veicolata da una prospettiva "liberale» che si definisce nei termini attualizzanti dell'opposizione dell'Atene termidoriana al laconismo montagnardo. La contrapposizione dell'Atene liberale alla Sparta guerriera nasce, cioè, come elemento di un dibattito interno alla cultura francese negli anni a cavallo tra il $1794 \mathrm{e}$ il 1795. Con la caduta del regime montagnardo, tuttavia, la tendenza a contrapporre le caratteristiche statuali delle «repubbliche» antiche non si ferma affatto e prosegue anzi con maggior fervore anche quando l'incubo del Terrore è ormai lontano. La pagina del diario di Constant da cui siamo partiti reca la data del maggio 1804; gli Études d'histoire ancienne sono del 1811. Occorre pertanto chiedersi quale senso assuma l'antitesi Sparta/Atene agli inizi dell'Ottocento e quali eventi continuino ad alimentare il dibattito sui modelli repubblicani antichi.

Nell'ormai famosa pagina del Journal intime, Constant attribuisce «idee spartane», confrontabili con quelle di Robespierre e seguaci, al paradigma statuale tratteggiato da Fichte. L'analisi di Constant è sintomatica dell'avvio di un nuovo processo. Col

15 C. Ampolo, "Per una storia delle storie greche», in SetTis (a cura di), I Greci, p. 1062; NIPPEL, «Antike und moderne Freiheit», p. 57-59.

16 Cf. anche Constant, Della libertà degli antichi paragonata a quella dei moderni, p. 222: «Fra tutti gli Stati antichi Atene è quello che più somigliò agli Stati moderni», p. 225: «Il commercio aveva fatto scomparire da Atene molte delle differenze che distinguono i popoli antichi dai popoli moderni.»

17 Canfora, Ideologie del Classicismo, p. 12. 
profilarsi dello scontro franco-prussiano agli inizi dell'Ottocento, gli intellettuali francesi rifunzionalizzano l'immagine "conventuale» di Sparta, elaborata in opposizione al regime montagnardo, trasferendola nel contesto del dibattito anti-prussiano. Nata come strumento di lotta politica interna, l'opposizione Atene liberale/Sparta guerriera diviene elemento centrale di una polemica rivolta verso l'esterno, in cui i termini dell'opposizione mascherano la rivalità politica e culturale tra la potenza francese e quella prussiana. In altri termini, i pensatori francesi tendono a rappresentare lo Stato prussiano alla luce del modello spartano elaborato in opposizione alla dittatura giacobina. Mentre la rappresentazione in vesti «ateniesi» della Francia liberale resta costante ${ }^{18}$, ad indossare abiti «spartani» sono ora i nuovi nemici: non più i capi montagnardi ma i Prussiani.

L'ipotesi che la cultura liberale francese di fine Settecento/inizio Ottocento usi il modello spartano per mettere in valore le coordinate semantiche del «nemico politico» trova conferma nella formazione culturale e nelle esperienze biografiche dello stesso responsabile dell'ideazione dell'immagine guerriera e rigoristica di Sparta. All'interno della cultura francese di inizio Ottocento, Constant si distingue per la sua conoscenza diretta della nuova «Scienza tedesca dell'Antichità»: conoscenza maturata attraverso ripetuti soggiorni a Gottinga, la lettura di Wolf e Creuzer, i rapporti personali con Wilhelm von Schlegel. Siffatte esperienze suggeriscono di esaminare la possibilità che Constant abbia elaborato la categoria di «idee spartane» obbedendo a un duplice impulso: non solo trasferendo sulla Prussia l'immagine spartana della dittatura montagnarda, ma reagendo anche all'immagine di Sparta che in quegli stessi anni matura in ambito prussiano per opera di Friedrich von Schlegel, fratello di quel Wilhelm che Constant conobbe personalmente entro la cerchia di Madame de Staël ${ }^{19}$.

Nel 1794 Friedrich von Schlegel scrive un famoso saggio Von den Schulen der Griechischen Poesie ${ }^{20}$. L'autore classifica le opere poetiche dell'antica Grecia dividendole in «scuole», corrispondenti a vari gruppi etnici (Stämme). Questi ultimi esprimono se stessi nelle forme politiche, nella lingua, nell'arte, nei costumi, nelle tradizioni mitologiche, distinguendosi nettamente gli uni dagli altri. Ioni e Dori formano i raggruppamenti più importanti, seguiti da Eoli e Alessandrini. Ma nel sistema teorico di Schlegel spicca su tutti il ruolo dei Dori, che l'autore considera, a dispetto degli Ioni «orientalizzati», come il «ramo» greco più antico e autentico. Schlegel sostiene che i Dori avrebbero diffuso le arti della musica e della ginnastica, sviluppando quell'ideale di bellezza in seguito esteso all'intero mondo greco; sottolinea l'importanza attribuita nelle società doriche all'educazione (non solo degli uomini ma anche delle donne), al rispetto della legge e

18 Cf. in particolare N. LORAuX, «La démocratie à l'épreuve de l'étranger (Athènes-Paris)», in Droit (éd.), Les Grecs, les Romains et nous, p. 164-190; P. VIDAL-NaqueT, «Parigi-Atene e ritorno", in ID., La democrazia greca nell'immaginario dei moderni, p. 247-253.

19 Sui rapporti di Constant con la cultura tedesca, cf. VIDAL-NAQUET, «La Grecia nell'immaginario degli uomini della Rivoluzione», p. 236-237.

20 Berlinische Monatsschrift 1794, November, p. 378-400. Cf. Rawson, The Spartan Tradition in European Thought, p. 318-320. 
al sentimento della libertà; ammira la capacità dei Dori di costituire comunità politiche più stabili e durature di quelle ioniche, che ben presto persero la libertà e conobbero i mali della tirannide e dell'anarchia. Al cospetto dell'ambiente dorico (Sparta), il mondo ionico (Atene) fa una magra figura. Esposte agli influssi dell'Oriente per via di intensi traffici commerciali, le città ioniche non seppero conservare quella "grecità» autentica che compete unicamente al mondo dorico. L'attività mercantile, in cui Constant riconosce la «modernità» di Atene, è in Schlegel responsabile della deriva orientalizzante della città. Al contrario Sparta, il convento guerriero ed egalitario di Constant, è in Schlegel sinonimo di libertà, stabilità, arte, bellezza. Questo sistema di opposizioni non è casuale. L'antitesi costante delle posizioni espresse da Schlegel e Constant testimonia l'esistenza di un acceso dibattito franco-prussiano sui modelli «repubblicani» antichi.

Il dibattito si fa più chiaro ed esplicito nell'opera di August Boeckh (17851867). Nella Staatshaushaltung der Athener (1817), l'autore ricostruisce i meccanismi di funzionamento dello Stato ateniese attraverso un'attenta analisi delle entrate e delle uscite e dei vari organi preposti alla loro gestione. Inserito nel contesto politicoculturale contemporaneo, lo studio di Boeckh assume uno spiccato valore polemico: «Soltanto l'unilateralità o la superficialità scorge ovunque l'ideale dell'antichità» e vede negli antichi gli unici compagni degni della propria «immaginaria grandezza ${ }^{21}$ ». Non ci sono dubbi su chi incarni, nella prospettiva dello storico prussiano, il suddetto atteggiamento di «unilateralità o superficialità». La critica di Boeckh è rivolta contro l'immagine idealizzata di Atene, diffusa nella cultura liberale francese. Mentre quest'ultima celebra l'Atene democratica e liberale, Boeckh manifesta una radicale avversione nei confronti del modello democratico. La democrazia è accusata di aver provocato il crollo dello Stato ateniese con una cattiva conduzione delle finanze. Non solo gli sprechi economici legati alla retribuzione delle magistrature e alle spese per abbellire la città avevano gravemente danneggiato le finanze pubbliche, ma i cittadini, abituati a contare sul sostegno della polis, si erano abbandonati all'ozio: «Il popolo ateniese» si era trasformato in "un tiranno ${ }^{22}$ ». A dispetto degli sprechi e degli abusi della democrazia ateniese, Boeckh ammira - sulla scia di Schlegel - la frugalità di tipo spartano. La condanna di Atene e della gestione democratica delle finanze, l'elogio di Sparta e le critiche alla «tirannide» popolare lasciano trasparire l'opposizione prussiano-laconica al modello borghese-liberale e alla Francia post-rivoluzionaria.

Ancor più marcata è l'influenza delle idee di Schlegel e della prospettiva anti-francese nel pensiero e nell'opera di Karl Otfried Müller (1797-1840). Professore di filologia classica a Gottinga, Müller è autore di una Geschichte hellenischer Stämme und Städte, il cui primo volume (Breslau, 1820) è dedicato a Orchomenos und die Minyer, il secondo e il terzo (Breslau, 1824) a Die Dorier. Il concetto di Stämme deriva - come è evidente - da

21 Su Boeckh, cf. Ampolo, «Per una storia delle storie greche», in Seтtis (a cura di), I Greci, p. 1044.

22 A. Bовскн, Die Staatshaushaltung der Athener, I, Berlin, de Gruyter, 1967³ , p. 226. 
Schlegel, ma ad esso Müller conferisce una più spiccata connotazione «razziale», facendo dell'idea di stirpi «la base incrollabile per la ricostruzione della storia più antica ${ }^{23}$ ". In una lettera a Tieck, datata 12 aprile 1821, è lo stesso Müller che rivela all'amico il progetto di una «storia» della stirpe dorica: «Dell'evoluzione del carattere dorico per motivi più profondi, su cui si sono espressi Schlegel e Schleiermacher, si occupino altri; io rimarrò in quelle regioni della storia, in cui ci si accontenta di lasciare indiscussa la nazionalità quale condizione d'origine divina ${ }^{24}$.» Troviamo qui espressa l'idea portante del programma storiografico di Müller: una storia greca organizzata per stirpi o razze intese come unità con caratteri stabili. Dopo aver ribadito nella prefazione ai Dori che le nazioni hanno un "carattere determinato fin dall'inizio da una natura superiore», Müller riconosce la «natura spirituale» della «nazione» dorica in «una disposizione morale alacre, severa e rigorosa», che si riflette, in campo artistico, in ideali di «misura, moderazione e ordine»e, in campo politico, in valori quali l'obbedienza (Gehörsam), la soggezione (Scheu), il rispetto (Ehrfurcht) nei confronti della tradizione ${ }^{25}$.

Sono simili valori che hanno consentito alla costituzione spartana, la costituzione dorica per eccellenza, di durare più a lungo di tutte e di resistere meglio di qualsiasi altra a rivoluzioni e trasformazioni. Come Schlegel, Müller apprezza la stabilità delle istituzioni politiche doriche, ma la sua ammirazione si estende ad altri due aspetti, per noi di estrema importanza: l'autosufficienza economica e «l'aspirazione all'unità». Economicamente auto-sufficiente, Sparta ha conservato pressoché intatte le più antiche istituzioni e tradizioni elleniche, in quanto non ha sentito la necessità di scambi e rapporti con l'esterno. Al contrario gli Ioni, per natura aperti alle innovazioni e ricettivi nei confronti degli stimoli «altri», hanno ben presto contaminato se stessi e la propria lingua «nel molle clima d'Asia», andando incontro a «rammollimento e dege-

23 U. vON Wilamowitz Moellendorff, Storia della filologia classica, trad. it. Torino, Einaudi, 1967, p. 114. Lo studioso aggiunge che nella sua opera storica Müller non era giunto «al soggetto più importante, agli Ioni e agli Ateniesi che pertanto sottovalutava».

24 Sulla concezione mülleriana della storia, cf. A. WitTenburg, «I Dori di K.O. Müller», Annali della Scuola Normale Superiore di Pisa 14 (1984), p. 1038-1039; Ampolo, «Per una storia delle storie greche», in SETTIS (a cura di), I Greci, p. 1045-1047; J.H. BLoK, " "Romantische Poesie, Naturphilosophie, Konstruktion der Geschichte": Karl Otfried Müller's Understanding of History and Myth», in W.M. Calder III - R. SChlesier (Hrsg.), Zwischen Rationalismus und Romantik: Karl Otfried Müller und die antike Kultur, Hildesheim, Weidmann, 1998, p. 55-97. Per la ricostruzione delle fasi più antiche della storia greca Müller attinse largamente al patrimonio mitico, convinto che i racconti della tradizione celassero sotto gli orpelli della fantasia e dell'immaginazione un nucleo autentico di verità (cf. A. Momigliano, «K.O. Müller's Prolegomena zu einer wissenschaftlichen Mythologie and the Meaning of Myth», Annali della Scuola Normale Superiore di Pisa 13 [1983], p. 673-689).

25 K.O. Müller, Die Dorier, II, Breslau, J. Max und komp., $1844^{2}$, p. 58 e 372. 
nerazione ${ }^{26}$ ». Non siamo molto lontani dagli Ioni orientalizzati di Schlegel, ma ciò che distingue l'analisi di Müller è l'accentuazione - sulla scia del maestro Boeckh - di un secondo fattore di degenerazione: la democrazia. Ad Atene, l'avvento della democrazia aveva ridimensionato il potere delle antiche istituzioni aristocratiche di stampo dorico, diffuse in età arcaica in tutto il mondo greco, ma in seguito sopravvissute solo a Creta e a Sparta. Prima dell'Atene democratica era esistita un'Atene aristocratica, in cui le funzioni di governo erano concentrate nelle mani dell'Areopago, responsabile della sorveglianza dei costumi e dell'amministrazione della giustizia. Ma il potere delle istituzioni aristocratiche si era in seguito indebolito. Prima la riforma di Clistene e poi le leggi di Efialte avevano privato l'Areopago del suo potere e delle sue prerogative in ambito giudiziario; infine, Pericle aveva distrutto gli ultimi resti delle istituzioni aristocratiche ${ }^{27}$. L'Atene delle origini era crollata sotto la spinta di masse popolari corrotte da demagoghi desiderosi di allontanare gli Ateniesi «dai sentieri dei costumi ereditati e tramandati dai padri». Poiché suddetti «costumi» corrispondono nel pensiero di Müller alle antiche istituzioni aristocratiche di stampo dorico, l'Atene arcaica di Müller si configura evidentemente come un' "Atene dorica", fondata sul rispetto religioso della tradizione e dell'autorità: un'Atene ben diversa da quella «democratica», più volte oggetto delle critiche dello storico ${ }^{28}$.

La condanna della democrazia, della riforma di Clistene e delle leggi di Efialte, consente di riconoscere nell'immagine «dorizzata» di Atene la risposta prussiana al mito dell'Atene «borghese», caro alla coeva storiografia francese ${ }^{29}$. Ma la democrazia non è l'unico punto su cui Müller polemizza con la scuola francese. Nell'opera dello storico

26 K.O. Müller, Die Dorier, I, p. 17. Sull'uso storiografico del concetto di «stirpi», cf. E. Will, Doriens et Ioniens. Essai sur la valeur du critère ethnique appliqué à l'étude de l'histoire et de la civilisation grecques, Paris, Les Belles Lettres, 1956.

27 K.O. Müller, Istoria della letteratura greca, II, trad. it. Firenze, Le Monnier, 1858, p. 11-19. Rispondendo all'accusa di sottovalutare il ruolo di Atene, rivoltagli da Schlosser in una recensione ai Dori (recensione apparsa su Heidelberger Jahrbücher 57 [1824], p. 898-927), Müller dichiara che la sua storia della stirpe dorica non implica la negazione del primato artistico e culturale di Atene (Prolegomena zu einer wissenschaftlichen Mythologie, Göttingen, Vandenhoeck und Ruprecht, 1825, p. 50). Sulla posizione di Müller nei confronti della letteratura attica, cf. R. Di Donato, «Una vera storia. La letteratura greca di K.O. Müller e la sua fortuna», Annali della Scuola Normale Superiore di Pisa 14 (1984), p. 1168-1170.

28 Cf. G. Cambiano, «L'Atene dorica di Karl Otfried Müller», Annali della Scuola Normale Superiore di Pisa 14 (1984), p. 1045-1067.

29 Si tratta di un processo di cui già i grandi storici dell'Ottocento ebbero piena consapevolezza. Nell'introduzione a La Cité antique (1864), Fustel de Coulanges scrive: «L'idea che ci si è fatta della Grecia e di Roma ha spesso turbato le nostre generazioni [...] I nostri ultimi ottant'anni hanno mostrato chiaramente che una delle grandi difficoltà che si oppongono al procedere della società moderna è l'abitudine presa di avere sempre davanti agli occhi l'antichità greca e romana» (N.D. Fustel DE COULANGES, La Cité antique, a cura di F. Hartog, Paris, Flammarion, 1984, p. 2). 
tedesco, inclinazione alle innovazioni e tendenza alle lotte intestine caratterizzano il mondo ionico quanto «l'aspirazione all'unità» distingue quello dorico. Si tratta dell'aspetto saliente e ideologicamente più connotato della stirpe dorica. Müller afferma che presso i Dori «l'aspirazione all'unità» fu più forte che presso tutte le altre nazioni. Per questo motivo, Sparta, modello della città dorica, è nello stesso tempo il modello esemplare di Stato, inteso da Müller come «unità riconosciuta dalla coscienza degli individui ${ }^{30}$ ». Facendo di Sparta un esempio di stabilità e di unità, Müller risponde alle critiche di area francese rivolte alla città laconica in nome di Atene. Come nota Giuseppe Cambiano ${ }^{31}$, la Sparta di Müller assume nell'Europa della Restaurazione una spiccata connotazione «reazionaria», configurandosi come modello di unità, autosufficienza economica, ordine sociale, resistenza alle rivoluzioni. Ma l'elogio dell'unità dorica rivela anche un altro evidente riferimento all'attualità. In quanto esempio di aspirazione all'unità, Sparta assurge al rango di modello per la Germania contemporanea, in cui «libertà significa $[. .$.$] cercare di disgregare lo Stato nelle sue componenti { }^{32}$ ».

A mo' di ricapitolazione, potremmo dire che, mentre nella storiografia francese l'opposizione Sparta/Atene assume le forme del conflitto tra «un'Atene borghese e letterata» e "una Sparta virtuosa e militare», nella storiografia prussiana l'antitesi tra i modelli repubblicani antichi si declina nei termini del conflitto tra un'Atene contaminata da influssi esterni e retta da demagoghi spreconi e una Sparta economicamente autosufficiente, culla dell'educazione e delle arti, promotrice di ideali di concordia e di unità. La radicale opposizione tra le rappresentazioni appena esaminate non deve né può stupire. La risemantizzazione delle categorie di "ateniese» e "spartano" segue da vicino gli eventi contemporanei, realizzandosi all'interno del dibattito culturale e del conflitto politico franco-prussiano. L'attualità politica del XIX secolo segna pesantemente i nascenti studi storici, comportando esiti che vanno ben oltre il panorama finora tracciato $^{33}$. La tendenza, comune alla nascente storiografia francese e tedesca, a interpretare le vicende antiche alla luce dei problemi attuali, determina, infatti, nei due diversi contesti nazionali non solo una differente rappresentazione dei modelli repubblicani antichi, ma anche una differente ricezione della storia greca e dell'opera di Tucidide in particolare.

Abbiamo visto come in Francia la riscoperta di Tucidide, all'indomani della caduta di Robespierre, sia associata alla lotta politica anti-montagnarda e all'affermarsi dell'ideologia borghese-liberale prima, alla polemica anti-prussiana poi. Nel periodo post-rivoluzionario, Tucidide sostituisce Plutarco nel ruolo di storico della libertà:

30 Müller, Die Dorier, II, p. 2. Sull'idea mülleriana di Stato, cf. Wittenburg, «I Dori di K.O. Müller», p. 1040-1041.

31 Cambiano, «L'Atene dorica di Karl Otfried Müller», p. 1049.

32 Müller, Die Dorier, II, p. 2.

33 Sull'influsso del presente nella ricostruzione degli eventi del passato, cf. F. Hartog, Régimes d'historicité. Présentisme et expériences du temps, Paris, Le Seuil, 2003. 
una libertà certo lontana da quella dei moderni (Constant), ma pur sempre funzionale all'opposizione liberale nei confronti del laconismo montagnardo e prussiano. Gli intellettuali prussiani, dal canto loro, riscoprono Tucidide - come dimostrano gli studi tuttora in corso di Payen - nel periodo immediatamente successivo all'occupazione napoleonica di Berlino e ne fanno il modello della nascente storiografia tedesca in opposizione alla cultura degli invasori francesi. Nella Prussia appena liberata dagli eserciti d'Oltralpe, Tucidide diviene modello della storia intesa come conoscenza e agire politico: il modello attraverso cui leggere gli eventi contemporanei ed esprimere l'ansia nazionale di rivalsa e di ricostruzione ${ }^{34}$. In altri termini, la ricezione dell'opera tucididea e della storia greca più in generale muta nella misura in cui essa è chiamata a rappresentare diversi stimoli e rivendicazioni particolari. Ne abbiamo una prova fondamentale nell'ambito della valutazione della Guerra del Peloponneso e degli eventi successivi a Cheronea. Partiamo ancora dalla Francia.

L'Atene borghese degli intellettuali termidoriani trova la sua consacrazione definitiva nell'opera di Victor Duruy (1811-1894), autore di una Histoire grecque (1851) destinata a esercitare un enorme influsso sull'insegnamento della storia in Francia. L'opera è costruita attorno all'antitesi Atene/Sparta. Duruy porta alle estreme conseguenze l'opposizione, già istituita da Diderot, tra la produttività di Atene e la sterilità di Sparta ${ }^{35}$. Alla città laconica, «macchina da guerra incapace di produrre», «strumento di distruzione che ha finito per distruggersi da sé», si contrappone la città attica, dotata delle «istituzioni più umane, più profondamente liberali che l'antichità abbia mai conosciuto»: le istituzioni democratiche. Se la Grecia antica è «la scuola del mondo», essa lo deve ad Atene, per cui Duruy dichiara apertamente la sua «simpatia». Lo storico elogia la democrazia ateniese, fondata sulla libertà, il commercio, le arti, la colonizzazione, la proprietà, la famiglia; descrive con entusiasmo la "politica sociale» dell'Atene periclea («questa età dell'oro dello spirito umano») e tesse l'encomio di Efialte, grazie a cui gli Ateniesi «hanno bevuto a lunghi sorsi alla coppa della libertà ${ }^{36}$ ». Il mito dell'Atene borghese, vicina allo "spirito di commercio» della Parigi contemporanea, si lega nell'opera di Duruy alla condanna delle leggi di Licurgo, considerate inapplicabili e immorali. Nella prospettiva dello storico, la vittoria di Sparta nella Guerra del

34 Occorre tuttavia ricordare che il laconismo prussiano conobbe anche alcune significative eccezioni. Per esempio, Ernst Curtius (1814-1896), allievo di Müller e autore di una Storia greca fino al 338, mostra nella sua opera una spiccata simpatia verso gli Ioni e gli Ateniesi.

35 Nell'articolo sul «Lusso» del Dictionnaire philosophique (1764), Diderot nota che Sparta, a differenza di Atene, non giovò per nulla ai suoi vicini né produsse grandi uomini. Ispirato a un evidente anti-laconismo è anche l'articolo dell' Enciclopedia dedicato a «Greci, filosofia dei», in cui Diderot, passando in rassegna i legislatori classici, accusa Licurgo di aver istituito un regime simile a un monastero e di aver promulgato leggi «incompatibili con un grande Stato e uno Stato commerciale».

36 Loraux, Vidal-NaQUeT, «La formazione dell'Atene borghese», p. 166-171; 200-204. 
Peloponneso assume connotati sinistri, segnando l'inizio della decadenza greca e la crisi delle istituzioni democratiche. Una condanna altrettanto dura coinvolge la conquista di Alessandro e l'età dei Diadochi: l'unica cosa che Alessandro donò al mondo sottomesso fu -afferma Duruy- «l'uniformità della schiavitù ${ }^{37}$. Ancora una volta, l'analisi dello storico francese sembra proporsi come contro-risposta nei confronti della storiografia prussiana e dell'opera di Johann Gustav Droysen in particolare. Figlio di un cappellano militare e allievo di Boeckh, Droysen (1808-1884) matura dalla tradizione familiare e dalle esperienze della sua formazione culturale uno spiccato spirito filo-prussiano. Fautore della creazione di uno Stato tedesco unitario sotto la guida della Prussia (prussianesimo), Droysen partecipa come membro del Parlamento di Francoforte all'elaborazione di una nuova costituzione per la Confederazione germanica ${ }^{38}$. La riunione dell'Assemblea nazionale tedesca segue immediatamente i moti rivoluzionari del 1848 e pone fine alle rivendicazioni autonomiste dei Länder. Non c'è episodio più importante per comprendere il senso dell'impegno politico e dell'attività storiografica di Droysen. Nella prospettiva dello storico prussiano, la partecipazione al progetto di una Germania unificata fa tutt'uno con la riscoperta e la rivalutazione di un periodo storico, quello di Alessandro Magno e dei Diadochi, in cui si affaccia per la prima volta nella storia antica il tema dell'unità politica della Grecia ${ }^{39}$. È da questa riflessione che nasce la Geschichte des Hellenismus (1836-1843), preceduta nel 1833 dalla pubblicazione della Geschichte Alexanders des Grossen.

37 Sulla visione storica di Duruy, cf. P. PAYEN, «Conquête et influences culturelles. Écrire l'histoire de l'époque hellénistique au XIX siècle (Allemagne, Angleterre, France)», Dialogues d'Histoire Ancienne 34 (2008), p. 123-128. Una prospettiva analoga si ritrova nella Storia greca di Gustave Glotz (1862-1935). L'autore considera il 404 come l'inizio della decadenza, di uno stato permanente di discordia e di crisi delle istituzioni. La lettura anti-spartana e anti-macedone della storia successiva a Egospotami e Cheronea caratterizza già il Voyage du jeune Anacharsis en Grèce di Jean-Jacques Barthélemy, testo pubblicato per la prima volta nel 1788 ma oggetto di numerose ristampe durante tutto il XIX secolo. Su Barthélemy, cf. P. Vidal-NaqUeT, «La démocratie athénienne en 1788», in E. GreCo (a cura di), Venticinque secoli dopo l'invenzione della democrazia, Paestum, Edizioni Fondazione Paestum, 1998, p. 155-161.

38 P. PAYEN, "Johann Gustav Droysen et l'Histoire de l'Hellénisme. L'époque hellénistique entre Alexandre et la Prusse», in J.G. Droysen, Histoire de l'Hellénisme, Grenoble, Jérôme Millon, 2005; ID., "L'Histoire de l'Hellénisme de Johann Gustav Droysen à travers sa correspondance: entre science et prussianisme», in C. BONNET - V. KRINGS (éd.), S'écrire et écrire sur l'Antiquité. L'apport des correspondances à l'histoire des travaux scientifiques, Grenoble, Jérôme Million, 2008, p. 139-153.

39 Droysen è tradizionalmente ritenuto il primo studioso ad aver intrapreso uno studio storiografico del periodo ellenistico. Le lezioni sull'età ellenistica tenute da Niebuhr all'Università di Bonn (1826; 1829-1830) furono, infatti, pubblicate solo nel 1851. Con buoni argomenti, Luciano Canfora (Ellenismo, Roma-Bari, Laterza, 1987) riconosce in Niebuhr il precursore del concetto droyseniano di Ellenismo, inteso come mescolanza e assimilazione. 
Abbiamo già visto come l'unità assicurata al mondo greco dalla conquista di Alessandro sia interpretata da Duruy in termini di «uniformità della schiavitù». La prospettiva filo-prussiana di Droysen comporta naturalmente un'interpretazione di segno opposto. Contro i particolarismi delle città greche e la visione ristretta di Demostene, l'autore elogia il progetto panellenico di Alessandro e l'unità politica assicurata dal sovrano macedone e dai suoi successori. Droysen vede in Alessandro la figura dell'uomo politico che si è posto il problema «attuale» dell'unità; lo considera il creatore di una "civiltà cosmopolita», nata dalla «fusione» fra Oriente e Occidente ${ }^{40}$. Il concetto di Ellenismo, inteso come mescolanza e assimilazione, rovescia la visione fichtiana e mülleriana dello Stato commerciale chiuso, economicamente auto-sufficiente, facendo della conquista di Alessandro il grande modello per l'unificazione della Germania. La rivalutazione in chiave positiva ed unitaria della "mescolanza» e della "fusione» non compromette tuttavia il tradizionale laconismo prussiano. Il filomacedonismo trova anzi il suo fondamento ideologico proprio nel filolaconismo. Come ha dimostrato Arnaldo Momigliano, i destini di Sparta e della Macedonia si erano del resto già incontrati nell'opera dello stesso creatore del mito sparto-prussiano. Per l'edizione inglese dei Dori Müller aveva scritto un saggio sulle sedi originarie dei Macedoni, stabilendo così un legame diretto tra la storia macedone e la stirpe dorica, da un lato, il progetto prussiano di unificazione nazionale, dall'altro ${ }^{41}$.

In modo ancora più esplicito, il tentativo di Sparta di unire la Grecia e quello prussiano di unire la Germania sono pensati in un rapporto "analogico» di continuità nella Habilitationsschrift («tesi di abilitazione») di Georg Busolt, intitolata Die Lakedämonier und ihre Bundesgenossen e pubblicata a Leipzig nel $1878^{42}$. Siamo all'indomani della Guerra franco-prussiana (1870-1871), il conflitto che porta a compimento i sogni di rivalsa nutriti per decenni dalla Prussia all'indomani del durissimo «schiaffo» dell'occupazione napoleonica. La vittoria riportata nella guerra del 1870-1871 ripaga finalmente i Prussiani del grave torto del 1806 e non manca di ripercuotersi sull'analisi storica di Busolt. Lo storico confronta la situazione della Germania prima della Guerra francoprussiana con quella della Grecia prima delle Guerre Persiane (p. 248). In entrambi i casi, gli unici momenti di unità prima delle grandi guerre «nazionali» sarebbero stati rappresentati da occasioni festive comuni; in seguito uno Stato militarista, Sparta/ Prussia, avrebbe imposto un sistema federale «unificante». Non si può non notare come

40 Cf. P. Payen, «L'Orient au laboratoire de l'hellénisme. I. Droysen», Archiv für Religiongeschichte 8 (2006), p. 161-178; ID., «El cosmopolitismo de las naciones en la Historia del Helenismo (1877-1878) de Johann Gustav Droysen», Revista de Historiografia 4/1 (2007), p. 84-91.

41 A. Momigliano, "Premesse per una discussione su K.O. Müller», Annali della Scuola Normale Superiore di Pisa 14 (1984), p. 899.

42 Sulla vita e sul pensiero di Busolt, cf. Rawson, The Spartan Tradition in European Thought, p. 326-329; M.H. Chambers, Georg Busolt. His Career in his Letters, Leiden, Brill, 1990, p. 11-44. 
nell'analisi delle Guerre Persiane Busolt enfatizzi l'importanza di Sparta, mettendo in secondo piano il ruolo di Atene: quell'Atene che solo due anni prima (1876) Ernest Renan (1823-1892) aveva considerato, nella celebre Prière sur l'Acropole, come la vera protagonista del «miracolo greco».

L'impressione che il conflitto franco-prussiano si celi ancora sotto la maschera dell'antitesi Sparta/Atene è forte, ma l'apparenza non deve ingannare. Busolt si colloca al termine di un processo durato più di settanta anni e non a caso, infatti, abbiamo scelto la data di pubblicazione della sua Habilitationsschrift come termine temporale estremo del nostro lavoro ${ }^{43}$. L'opera dello storico prussiano rappresenta una frattura chiara rispetto alla tradizione ottocentesca. A differenza dei suoi predecessori, Busolt non guarda tanto a Tucidide quanto ad Erodoto. Il filtro che lo storico prussiano sceglie per leggere e interpretare le vicende contemporanee non è più la Guerra del Peloponneso, ma le Guerre Persiane. Busolt vede nei Greci/Spartani l'azione vittoriosa ed unificante dello Stato prussiano, mentre riconosce nella Persia baldanzosa e infine sconfitta l'immagine della Francia post-napoleonica. Stranezze della storia: da «ateniesi», i Francesi sono ora divenuti «persiani». La storia - si sa - è a volte davvero beffarda. Non solo i «Francesi» di Busolt, ma anche Müller ne fece esperienza. Il creatore del mito dorico morì a causa di un malore improvviso ad Atene, nel corso di un viaggio in Grecia. Aveva solo 42 anni. Il mito da lui creato visse invece - e con tristi conseguenze - decisamente più a lungo ${ }^{44}$.

Carmine PISANO
Dipartimento di Discipline Storiche «Ettore Lepore»
Università degli Studi di Napoli Federico II
Via Marina, 33
80133-Napoli
pisano.carmine@virgilio.it

43 La concorrenza scientifica tra Francesi e Tedeschi continua anche dopo la guerra franco-prussiana. Fustel de Coulanges polemizza con Theodore Mommsen in una serie di articoli, in seguito editi sotto il titolo di Questioni Contemporanee. Nelle Études sur la proprieté à Sparte (1888), Fustel confuta la tesi del primitivo comunismo spartano, mentre in La Monarchia Franca (secondo volume della monumentale Storia delle istituzioni politiche dell'antica Francia) dimostra che le istituzioni francesi non hanno nulla in comune con il diritto tedesco. Se, da parte tedesca, Engelbert Drerup (1871-1942) pubblica una caricatura di Atene e della Francia, descritta come «una repubblica di avvocati», da parte francese, Georges Clemenceau (1841-1929) inneggia a Démosthène et la liberté grecque. Cf. P. VidAl-NaQueT, «En guise d'introduction: une Grèce à la française?», in ID., Les Grecs, les historiens, la démocratie, p. 15-16; F. HARTOG, Le XIX siècle et l'histoire. Le cas Fustel de Coulanges (1988), Paris, Le Seuil, 2001, p. 398-404.

44 Nella cultura tedesca, il «miraggio spartano» continua fino a Hitler e all'affermazione del nazional-socialismo (cf. P. VIDAL-NAQUET, «Une invention grecque: la démocratie», in ID., Les Grecs, les historiens, la démocratie, p. 180). 